International Journal of Computer Networks \& Communications (IJCNC) Vol.9, No.6, November 2017

\title{
EWER: “ElECTROMAGNETIC WAVE EXPOSURE REDUCTION" SERVICE FOR SENSITIVE USERS With PRIVACY CONCERNS
}

\author{
Mohamed Aymen Chalouf ${ }^{1}$, Francine Krief ${ }^{2}$ and Dramane Ouattara ${ }^{2}$ \\ ${ }^{1}$ IRISA, University of Rennes 1, 6 rue Kerampont 22300 Lannion, France \\ ${ }^{2}$ LaBRI, University of Bordeaux, 351 cours de la Libération , 33400 Talence, France
}

\begin{abstract}
The impact of electromagnetic waves, and consequently of wireless and mobile transmissions, on human health represented an open debate for many years. That is why, for example, demonstrations take place regularly against the installation of new transmission antennas in urban areas. In this paper, we propose a service aiming at limiting the exposure level of individuals to electromagnetic radiation in some areas. This could be achieved thanks to a dynamic adaptation of transmission parameters (frequency, power, etc.), for example. The proposed service defines some components implementing the intelligence required to compute the exposure level of a person and to carry out the adapted actions. It also relies on a signalling protocol that integrates an "Electromagnetic Wave Exposure" component which groups the parameters required to manage the exposure level of sensitive persons. To our knowledge, there is no solution providing a similar service to reduce the exposure level of sensitive persons to electromagnetic radiation. Moreover, by controlling the electromagnetic radiation exposure level of individuals, our proposal may lead to the limitation of electromagnetic waves. This will also help to meet the energy-saving constraints that future networks, particularly 5G, should be able to meet.
\end{abstract}

\section{KEYWORDS}

Sensitive persons, Electromagnetic radiation (EMR), EMR exposure level, Wireless communications, Signalling protocol, Network auto-configuration.

\section{INTRODUCTION}

Today, we are invaded by equipment and devices that communicate via electromagnetic waves. This is due to the rapid evolution of wireless and mobile access technologies and their widespread deployment. This phenomenon will be accentuated by the arrival of the IoT (Internet of Things). Thus, connection density should be multiplied by 10 with the $5 \mathrm{G}$, i.e. 1 million devices / Km2 [1]. In such an environment, an important challenge will be the reduction of the electromagnetic radiation levels to allow a better cohabitation between the various wireless communication systems. This will result in reducing the Electro-Magnetic Radiation (EMR) exposure level of humans. This EMR reduction will contribute to the limitation of the energy consumed in wireless and mobile transmissions and, thus, the carbon footprint of ICT (Information and Communication Technologies). Indeed, currently, about 5\% of the world's energy consumption is due to ICT, which is comparable to the carbon footprint of the commercial air traffic and this consumption doesn't stop increasing [2]. Public health studies have focused on the impact of electromagnetic radiation on individuals. This Impact depends on several parameters such as frequencies and transmits powers. Several countries, e.g. France, are issuing recommendations to reduce the EMR exposure level of individuals. 
In this context, we propose a service that limits the exposure level of individuals to radio waves in some areas. This will be achieved through dynamic adaptations of the used bands and on-the-fly modification of transmissions parameters (e.g. transmit power).

To control the exposure level of individuals to electromagnetic waves, the service we propose (EWER: Electromagnetic Wave Exposition Reduction) will allow coordination between the various network actors in order to perform the following tasks: (1) compute / estimate the global exposure level of a sensitive person, act to minimise this level and alert the concerned person when it is needed. To do this, the key components of our service must implement modules for the management of the exposure level and use a signalling protocol in order to communicate with each other when necessary.

The reminder of this paper is organised as follow. In Section 2, we introduce the impact of electromagnetic radiation on sensitive persons as well as some protective measures. This section also describes the network context in which our service can act. In Section 3, we describe the signalling protocol that will be used by our service (EWER). Section 4 provides details on the components of the EWER service and how it operates. In Section 5, we show some scenarios to illustrate the utility of the proposed service and how it works: execution, computations, signalling, etc. Finally, Section 6 concludes the paper and describes some interesting perspectives.

\section{State Of The ART}

The electromagnetic wave exposure problem can be considered from different aspects: possible health effects [3,4], measuring wave exposure [5,6] and reducing electromagnetic radiation exposure $[7,8]$.

In [3] H.S. Aboul Ezz and al. study the undesired neurological effects of electromagnetic radiation exposure. They conclude that the electromagnetic radiation exposure may affect memory, learning, and may cause stress. In [4] Kavindra Kumar Kesari and al. conclude that radio frequency electromagnetic wave from commercially available cell phones might affect the fertilising potential of spermatozoa.

In [5] S. Mann presents body-worn instruments for measuring the strengths which can be used to assess personal exposures to environmental radio frequency electromagnetic fields. The European project LEXNET (Low EMF Exposure Future Networks) defines a more realistic human exposure index combining exposure induced by access points or base stations and the exposure induced by the devices (mobile, laptop) [6].

In [7] J. Palicot and C. Roland propose to use cognitive radio for decreasing electromagnetic radiation. In [8], Yusuf Abdulrahman Sambo and al. compile the most interesting results and ideas related to EMR exposure in mobile communication systems and present possible ways to reduce it. In particular, they identify five techniques for reducing electromagnetic radiation exposure: SAR shielding, power control, beamforming, CoMP, and massive MIMO.

The above-mentioned works address the electromagnetic wave exposure problem from different perspectives. However, this work is the first one to propose a service for reducing the exposure to the electromagnetic waves resulting from radio communications. Indeed, to our knowledge, there is no work that deals with the reduction of the individuals' exposure to radio communication waves in areas where several radio communication technologies coexist. Moreover, our approach is quite complete, and based on (1) smart components that will be implemented by the key 
network equipment and (2) a signalling protocol enabling them to co-ordinate their action for efficient and optimal results.

\subsection{EFFECT Of ELECTROMAgNETIC RAdiation ON INDIVIDUALS HEALTH}

Mobile phones, WiFi routers and other wireless devices are invading our daily lives. These devices become increasingly sophisticated every year and their use simplified. They all emit radiation called electromagnetic radiation or electromagnetic wave. Today, the question of the effects of electromagnetic radiation on people health arises. Research onto the operation of these devices does not provide a clear and precise answer to this concern. The question remains without an answer, whether concerning mobile telephony, relay antennas or microwaves. However, recommendations converge towards a reduction of the exposure level and its control in order to prevent possible health risks. To introduce and integrate this requirement into future network architectures, it is essential to start by identifying the metrics to control and the possible actions to perform, while identifying the persons having sensitivity to electromagnetic waves.

\subsubsection{Sensitive PeOPLE}

Some persons are more sensitive than others to electromagnetic waves. It is the case of children who, according to ANSES (French Agency for Food, Environmental and Occupational Health Safety), "must be considered as a population more exposed to radio frequency electromagnetic waves".

It is also the case of persons equipped with medical devices which could be external (e.g. pumps) or implanted (e.g. pacemakers, cardiac defibrillators, cochlear implants, cerebral implants, neuro stimulators, retina encoders, insulin pumps). Indeed, electromagnetic waves can alter the operation of medical devices, whether they are active (e.g. pacemakers) or passive (e.g. heart valve prostheses, artificial articulation), which could result in serious risks for the wearer's health. That is why a number of recommendations are emitted for medical devices holders. Thus, persons equipped with external / implanted cardiac pacemakers must, for example, avoid: leaving their mobile phones in a pocket near the medical device or staying close to an anti-theft store protection gates. Otherwise, the ANSI (American National Standard Institute) defined a standard for evaluating electromagnetic compatibility between a mobile phone and a hearing device (implanted or worn) [9]. Different cases of dysfunction due to such interference were observed (e.g. false alarm, drug injection pump stop). It should be noted that the number of persons equipped with medical devices will not stop growing. This is due to the ageing of the population and the fact that younger people are more and more equipped with such medical devices.

Some people are also sensitive to electromagnetic waves. For these persons, using DECT phones, mobile phones or smart phones, can cause pain and heat in the ear, and even headaches, tinnitus and dysaesthesia (tingling, burning) in the face or in the arm and hand holding the earpiece. While the use of computers or televisions can cause signs of intolerance, such as a headache, eye disorders, dysesthesia in the hand holding the mouse, chest pain, tinnitus, false vertigo and balance disturbance after a certain period of exposure [10].

However, the entire population is not spared. As a reminder, in May 2011, the World Health Organisation (WHO) classified electromagnetic radiofrequency waves as "potentially carcinogenic" [11]. And the law "on sobriety, transparency, information and consultation on exposure to electromagnetic waves" [12] reinforces the application of the principles of precaution and sobriety concerning the emission of electromagnetic waves (relay antennas, mobile phones, smartphones, internet boxes, etc.).

The service we propose will help sensitive people and will enable applying the precautionary principle because risk reduction is needed. This service will act on the electromagnetic waves 
generated by wireless and mobile communications by applying adaptations and improvements whenever possible. Before detailing our proposal, we describe the current protection measures that we classify into two categories: (1) regulatory measures and (2) measures that could be taken by individuals to reduce the impact of electromagnetic waves in their surrounding.

\subsubsection{Current Protection Measures}

\subsubsection{REgULATIONS}

It is known that the effects of EMR (ElectroMagnetic Radiation) on the health of people are related to the exposure level [13]. Therefore, in order to evaluate precisely our own exposure level, for preventive or curative reasons, the use of measuring instruments is necessary. It is not a matter of measuring electromagnetic waves on the entire spectrum because not all waves have necessarily adverse health effects. Indeed, two main classes of waves are today identified as problematic: low-frequency EMF (HV lines and $230 \mathrm{~V}$ distribution, home appliances, etc.) to which we have been exposed in recent decades, and pulsed microwaves (mobile phones, mobile relays, DECT, WiFi, WiMax, Bluetooth, etc.), whose explosion is recent [13]. Thresholds of exposure to low frequencies can be estimated by referring to habitat biology benchmarks [14]. Concerning pulsed microwaves, the most common applications are those of mobile telephony.

In France, the ANSES does not report any specific adverse effects following, for example, the deployment of the 4G network. However, the ANFR (French Agency of Frequencies) indicates that this deployment, overlapping with existing technologies $(2 \mathrm{G}$ and $3 \mathrm{G})$, should increase the average of the exposure level outdoor by $50 \%$. Based on technology type and its impact level on people, two categories could be distinguished. Equipment classified as near body (e.g. GSM mobile phone) and equipment away from the body (BTS antennas, microwave ovens, etc.). To avoid adverse effects of electromagnetic waves on people's health, there is a regulation on exposure thresholds for the different frequency ranges. In the range of radio frequencies, the maximum allowed exposure is between 28 and $61 \mathrm{~V} / \mathrm{m}$ (volts per meter), depending on the used technology (e.g. FM radio, TV, 2G, 3G and 4G). These thresholds have been set to protect users against the thermal effect of electromagnetic waves, which consists in an increase in tissue temperature. These thresholds are very high. Therefore, they are not surpassed in reality, while other potential effects are possible such as those discussed in Section 2.1.1. For this reason, a resolution of the Council of Europe (2011) recommends, especially, that "a preventive threshold for microwave long-term exposure levels not exceeding $0.6 \mathrm{~V} / \mathrm{m}$ " should be established. For a mobile phone base station, the entire body is exposed. The measurement parameter is the level of the electric field (E-field). Thresholds to not exceed are as follows:

- for a GSM 900 antenna: $41 \mathrm{~V} / \mathrm{m}$,

- for a GSM 1800 antenna: $58 \mathrm{~V} / \mathrm{m}$,

- for an UMTS antenna: $61 \mathrm{~V} / \mathrm{m}$,

- for WiFi and microwaves oven: $61 \mathrm{~V} / \mathrm{m}$,

- for FM radio: $28 \mathrm{~V} / \mathrm{m}$.

For a cellular or mobile phone, only part of the body is exposed to electromagnetic radiation. The measurement parameter is the power absorbed per mass unit of body tissue, expressed in Watts per kilogram (W/kg). It is more commonly referred to as SAR (Specific Absorption Rate). The regulatory limit value in France to not exceed for a mobile phone is $2 \mathrm{~W} / \mathrm{kg}$. The SAR depends on the phone model. It must be included in the user guide of the phone. Table 1 summarises the main characteristics (frequency, SAR, etc.) of the most common technologies that we consider in our proposal. Table 2 shows the E-field values depending on the transmit power and the distance (d). 
Table 1. Technologies, frequencies and SARs [13].

\begin{tabular}{|c|c|c|}
\hline Technology & Frequencies & Limit transmit power \\
\hline Bluetooth & $2400-2483,5 \mathrm{MHz}$ & $\begin{array}{l}\text { 2nd class }(2.5 \mathrm{~mW}), 100 \mathrm{~mW}<0.01 \mathrm{~W} / \mathrm{kg} \\
\text { (1 st class) }\end{array}$ \\
\hline ZigBee & $\begin{array}{l}868 \mathrm{MHz}, 1 \mathrm{GHz}, 2.4 \\
\mathrm{GHz}\end{array}$ & $\begin{array}{l}4 \mathrm{~mW} \text {, Version future }(20<0.08 \mathrm{~W} / \mathrm{kg} \text { (body) } \\
\mathrm{mW})\end{array}$ \\
\hline LoRaWAN & $\begin{array}{l}125 \mathrm{kHz}, \quad 250 \mathrm{kHz} \\
865 \mathrm{MHz}-868 \mathrm{Mhz}\end{array}$ & \begin{tabular}{l|l}
$0.12589 \mathrm{~W}$ & $<0.08 \mathrm{~W} / \mathrm{kg}$ (body) \\
& $<2 \mathrm{~W} / \mathrm{kg}$ (head, trunk)
\end{tabular} \\
\hline NFC & $13.56 \mathrm{MHz}$ & \begin{tabular}{|l|l}
$2 \mathrm{~W}$ & $<0.08 \mathrm{~W} / \mathrm{kg}$ (body) \\
& $<2 \mathrm{~W} / \mathrm{kg}$ (head, trunk)
\end{tabular} \\
\hline WiFi & $2,4 \mathrm{GHz}, 5 \mathrm{GHz}$ & $\begin{array}{l}2.4 \mathrm{GHz}(0.1 \mathrm{~W}), 5 \mathrm{GHz}(1<0.2 \mathrm{~W} / \mathrm{kg}, 0.1 \mathrm{~V} / \mathrm{m}(\mathrm{d}=5 \\
\mathrm{W})\end{array}$ \\
\hline GSM & $900 \mathrm{MHz}, 1800 \mathrm{MHz}$ & \begin{tabular}{|l}
$2 \mathrm{~W}$ (GSM 900), 1W (GSM) $\begin{array}{l}\text { GSM 900: } 0.603 \mathrm{~W} / \mathrm{kg}(41 \\
\text { 1800), 20-60 W (3G) }\end{array} \mid \begin{array}{l}\text { GSM }) ; \\
\text { V/m) }\end{array}$ \\
\end{tabular} \\
\hline MIMO & $5-6 \mathrm{GHz}$ & $\begin{array}{l}\text { U-NII-1 band (indoor): } 50<0.08 \mathrm{~W} / \mathrm{kg} \text { (body) } \\
\mathrm{mW} \\
\mathrm{U}-\mathrm{NII}-2 \mathrm{~A} \text { and U-NII-2C } \\
\text { (indoor or outdoor): } 250 \mathrm{~mW}\end{array}$ \\
\hline RFID & $\begin{array}{l}125 \mathrm{kHz}, 13.56 \mathrm{MHz}, \\
900 \mathrm{MHz}\end{array}$ & \begin{tabular}{l|l}
$2 \mathrm{~W}$ & $<2 \mathrm{~W} / \mathrm{kg}$
\end{tabular} \\
\hline LTE & $\begin{array}{l}700 \mathrm{MHz}, 800 \mathrm{MHz}, \\
1800 \quad \mathrm{MHz}, \\
\mathrm{MHz}\end{array}$ & $\begin{array}{l}\text { LTE 800: } 39 \mathrm{~V} / \mathrm{m} \\
\text { LTE 2600: } 61 \mathrm{~V} / \mathrm{m}\end{array}$ \\
\hline UMTS & $1900-2100 \mathrm{MHz}$ & $0.5 \mathrm{~W} / \mathrm{kg}(61 \mathrm{~V} / \mathrm{m})$ \\
\hline WiMax & $3.5 \mathrm{GHz}$ & $10 \mathrm{~V} / \mathrm{m}(\mathrm{d}=5 \mathrm{~m})$ \\
\hline
\end{tabular}

Table 2. E-field values according to transmit power and distance [14].

\begin{tabular}{|l|l|l|l|l|l|l|l|}
\hline Distance & $\mathbf{5 c m}$ & $\mathbf{1 0 c m}$ & $\mathbf{2 0 c m}$ & $\mathbf{5 0 c m}$ & $\mathbf{1 m}$ & $\mathbf{2 m}$ & $\mathbf{5 m}$ \\
\hline 3G correct reception $(0.1 \mathrm{~mW})$ & 1.10 & 0.55 & 0.27 & 0.11 & 0.05 & 0.03 & 0.01 \\
\hline Bluetooth $(1 \mathrm{~mW})$ & 3.46 & 1.73 & 0.86 & 0.34 & 0.17 & 0.08 & 0.03 \\
\hline WiFi maximum $(100 \mathrm{~mW})$ & 34.6 & 17.3 & 8.66 & 3.46 & 1.73 & 0.86 & 0.34 \\
\hline 3G maximum $(125 \mathrm{~mW})$ & 38.7 & 19.3 & 9.68 & 3.87 & 1.93 & 0.96 & 0.38 \\
\hline DECT maximum $(250 \mathrm{~mW})$ & 54.7 & 27.3 & 13.7 & 5.47 & 2.73 & 1.37 & 0.54 \\
\hline GSM 1800 maximum $(1 \mathrm{~W})$ & 109 & 54.7 & 27.3 & 10.9 & 5.47 & 2.73 & 1.09 \\
\hline GSM 900 maximum $(2 \mathrm{~W})$ & 155 & 77.4 & 38.7 & 15.5 & 7.74 & 3.87 & 1.55 \\
\hline
\end{tabular}

\subsubsection{INDIVIDUAL MEASURES [10]}

To protect themselves against adverse effects of electromagnetic waves, some measures could be carried out by sensitive persons in any place (home, work, etc.). These measures concern two types of wave sources: internal sources and external sources.

To mitigate the internal sources effects, it is recommended, for example, to:

- reduce the use of devices emitting radio frequencies and microwaves such as mobile phones (use when it is unavoidable, prefer SMS and opt for a phone with the lowest possible SAR) and DECT phones (replace by corded telephones); 
- rearrange the places so as to be far enough away from the essential appliances (refrigerators, washing machines, etc.) and the electrical installation whose shielding should be improved;

- Avoid the use of induction boards (return to gas or thermal furnace), microwave ovens, wake-up radios and low energy bulbs;

- Avoid the use of electromagnetic field amplifiers.

As for external sources, they are multiple. We find, for example, electrical transformers, high or very high voltage lines, neighbouring WiFi antennas, relay antennas (radio, television, GSM), etc. Measures to mitigate the adverse effects of these external electromagnetic waves are not simple. Indeed, it is necessary to transform the place (house, office, etc.) in Faraday cage, by the realisation of a shield, or to move to another place.

In this work, we focus on electromagnetic waves generated by radio communications. These waves can be generated by internal sources (smartphones, WiFi antennas, wireless sensors, etc.) or external sources (WiFi antennas of neighbours, relay antennas, etc.) and can contribute to the increase of the global exposure level of a person. We estimate that the global exposure index of a person is proportional to the sum of the SARs of the different electromagnetic waves impacting this person [9]. A more realistic human exposure index combining exposure induced by access points or base stations and the exposure induced by the devices (mobile, laptop) is proposed in [8]. To contribute to efforts aiming at protecting people from adverse effects of electromagnetic radiation, we propose a service that will minimise the exposure level of sensitive persons by acting on wireless and mobile communications whose waves could impact those persons. This service will be based on mechanisms that we detail in the rest of the paper. It should be noted that these mechanisms use two main parameters: the exposure level of the sensitive person and his tolerance threshold. The tolerance threshold of a person specifies its degree of sensitivity. It depends only on the person's profile (pathologies, pacemaker, intolerable frequencies, etc.).

\subsection{Network Context Of OUr Proposal}

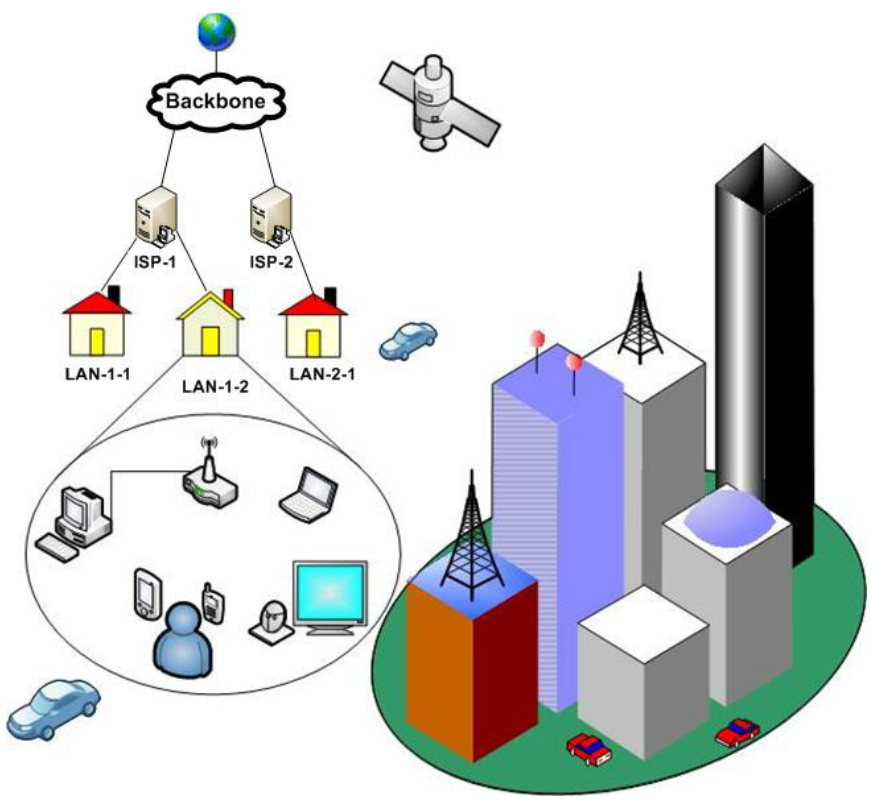

Figure 1. Architecture of the considered network context 
In the previous section, we have introduced the adverse effects of electromagnetic waves on the health of individuals, especially sensitive people. We also recalled some measures to protect them from the negative effects of some electromagnetic waves. Before describing our proposal aiming at reducing the effect of electromagnetic waves related to radio communications, we will describe the network context that we consider.

In order to guarantee better compatibility between our solution and the various current network architectures, we consider a general architecture where the different technologies, equipment and devices can coexist. In this architecture, we distinguish two main parts (see Figure 1).

- User's network: It is the network of the user. It may be composed of one or more small networks. These networks are generally interconnected with each other, and each of them is dedicated to specific applications or services. For example, a network of heterogeneous sensors equipping the user (e.g. WBSN: Wireless Body Sensor Network) or its environment (e.g. WSN: Wireless Sensor Network). To provide the user with a service, this network collects, stores and processes data. Thus, the intelligence required to implement the service is distributed on all network nodes. These nodes need to communicate with each other or with the outside. That's why an access point is often essential to provide the user with the needed services. Depending on the configuration of the user's network (nodes, architecture, etc.), the access point can be a smartphone (case of a WBSN equipping a mobile patient), a box (case of a home network) or an OBU (case of a VANET: Vehicular Ad-hoc NETwork).

- External network: This network is composed of all the other nodes and equipment (not included in the user's network) that could impact the global exposure index of the user. Thus, it includes the ISP's equipment (e.g. RRH, antenna relay, etc.) and eventually the nearby located LANs which can impact the exposure level of the considered person.

Communications in the user's network or with the outside can be based on different communication technologies (Ethernet, Wi-Fi, Bluetooth, UMTS, etc.). Table 3 summarises the main features of these two networks and the main used communications technologies.

Table 3. Characteristics and technologies of the considered network architecture.

\begin{tabular}{|l|l|l|l|}
\hline & Central node & Used technologies & Limits / Disadvantages \\
\hline $\begin{array}{l}\text { User's } \\
\text { network }\end{array}$ & $\begin{array}{l}\text { Smartphone, } \\
\text { Tablet, } \\
\text { HomeBox, } \\
\text { On-board } \\
\text { computer } \\
\text { (vehicle) }\end{array}$ & $\begin{array}{l}\text { Inside: Bluetooth, WiFi, ZigBee, } \\
\text { Radio-cognitive, etc. } \\
\text { With the outside: Cable, ADSL, } \\
\text { Fiber, WiMax, UMTS, LTE, etc. }\end{array}$ & $\begin{array}{l}\text { Capabilities: storage and } \\
\text { processing, Battery life, } \\
\text { Faraday cage phenomenon in } \\
\text { case of vehicle, Generated } \\
\text { electromagnetic waves, } \\
\text { Energy cost for mobile and } \\
\text { long-range wireless } \\
\text { communications }\end{array}$ \\
\hline $\begin{array}{l}\text { External } \\
\text { network }\end{array}$ & $\begin{array}{l}\text { ISP's Router, } \\
\text { DU Pool }\end{array}$ & $\begin{array}{l}\text { Inside: Bluetooth, Infrared, } \\
\text { Zigbee, Radio-cognitive, WiFi, } \\
\text { WiMax, UMTS, LTE, Cable, } \\
\text { Fiber, etc. }\end{array}$ & $\begin{array}{l}\text { Generated electromagnetic } \\
\text { waves, Energy cost for mobile } \\
\text { and long-range wireless } \\
\text { communications }\end{array}$ \\
\hline
\end{tabular}




\section{Proposed Signalling}

\subsection{NeEdS ANd PRinCiPLes}

The service we propose will be activated following the request of the user. Our service (EWER: Electromagnetic Wave Exposure Reduction) will allow acting on the sources of electromagnetic waves related to mobile and wireless communications. Depending on the overall context of the mobile / wireless communications in the user environment, the execution of the EWER service aims at reducing the exposure level of a sensitive person and may require organisation in:

- User's network, i.e. between the different equipment and nodes composing the user's network. For example, the nodes of a WBSN or those of the Home Network can be reorganised / reconfigured in order to reduce the impact of their radio transmissions on the sensitive user. In this case, we have what we call a « local action » that may be sufficient.

- External network, in this case an organisation with other wireless local networks (neighbouring or remote) or with the ISP is needed. This is essential when external network equipment (e.g. RRH: Remote Radio Head) affect the sensitive person. This is also the case when communications in the neighbouring wireless networks have an impact on the sensitive person or when user's network equipment are involved in service provision for remote users and generate waves to which the sensitive user is exposed.

When the exposure reduction requires local actions, the local component of the EWER service (EWEM-LM: Electromagnetic Wave Exposition Management - Local Module), located at the user's network access point (e.g. Smartphone, HomeBox), will make the best decision to reduce the exposure level (see section 4). This decision is based on a good knowledge of the whole user's network (devices, communications, waves, etc.) and the location of the sensitive person.

When reducing the exposure index of the user requires action from the external network, the central node of the user's network will ask the ISP to act in order to reduce the exposure index of the sensitive person. A signalling protocol is then required. We propose to use a signalling protocol which will allow the user's network to communicate with the external network in order to optimise their action aiming at minimising the exposure level of a sensitive person. In the next section, we will see the context of use of this signalling protocol.

\subsection{Signalling Protocol}

This signalling can be achieved using the Service Level Negotiation Protocol (SLNP) which we have defined and implemented in order to negotiate communications QoS (Quality of Service) and security in NGN (Next Generation Networks) [15]. The main advantage of this protocol is the use of Web Services which allows: (i) the interoperability between the different actors and (ii) the extension of the negotiated parameters. So, this protocol can easily be extended in order to take into account the impact of electromagnetic waves related to mobile and wireless communications on a sensitive person. In this work, we will focus on the use of SLNP in (i) the computation of the impact of electromagnetic waves on a sensitive person and (ii) the reduction of this impact.

\subsubsection{Messages}

The SLNP protocol is used to negotiate SLS (Service Level Specification: SLA technical parameters) for ISP's clients [15]. These negotiations may involve the different AS (Autonomous Systems) implied in data transport. To perform this negotiation, the SLNP protocol uses the six messages summarised in Table 4. 
Table 4. Messages used with SLNP

\begin{tabular}{|l|l|}
\hline Message & Role \\
\hline Negotiate & Request the establishment of one SLS (parameters and their values) \\
\hline Modify & Request the modification of an established SLS \\
\hline Release & Request the termination of an established SLS \\
\hline Revision & Propose an alternative to the requested SLS (Negotiate or Modify) \\
\hline Notify & Request the improvement / degradation of an established SLS \\
\hline \multirow{2}{*}{ Response } & Accept (Ack) or reject (Nack) a request (Negotiate, Modify or Release) \\
\cline { 2 - 2 } & Accept or reject an alternative (Revision) \\
\cline { 2 - 2 } & Accept or reject a notification \\
\hline
\end{tabular}

\subsubsection{PARAMETERS}

These messages include the parameters negotiated by the different actors and cover QoS and security [15]. In order to take into account the impact of mobile and wireless communications on a sensitive person, we integrate new parameters into the SLS. These parameters are essential to ensure a minimum or at least reasonable level of exposure (below the person's tolerance threshold).

The parameters that we define to enable the management of the electromagnetic wave exposure form the «EWE: Electromagnetic Wave Exposure » component of the SLS and include:

- State: state of the EWER service (activated or not). This ensures the proper functioning of the QoS and security negotiation when the electromagnetic radiation exposure is not considered.

- ExternalImpact: an estimation of the proportion of the non-local equipment's impact in the overall exposure index of the sensitive person. This proportion can be obtained in two ways, depending on whether the sensitive person is equipped with a device for continuous measurement of his exposure level or not. If it is the case, then this proportion (ExternalImpact) is calculated on the basis of the measured global exposure index and an estimation of the local equipment's impact (InternalImpact). As previously introduced, the impact of local equipment is calculated using the local component of the EWER service (EWEM-LM). However, if this type of measuring device is not available, then the external component of the EWER service (EWEMEM: Electromagnetic Wave Exposition Management - External Module), located at the ISP equipment, will compute this proportion. In the presence of a measuring device:

\section{ExternalImpact $=$ MeasuredGlobalExposureIndex - InternalImpact}

The global exposure index is proportional to the sum of the SARs of the electromagnetic waves impacting the considered person [9]. The SAR of an electromagnetic wave is equal to [16]: $S A R=\sigma[E]^{2} / 2 \rho$

where $\sigma$ is the conductivity of tissue simulant $(\mathrm{s} / \mathrm{cm}),[E]^{2}$ is the electric field strength $(\mathrm{V} 2 / \mathrm{cm} 2)$ and $\rho$ is the density of tissue simulant $(\mathrm{g} / \mathrm{cm} 3)$

- ExternalImpactThreshold: It is the threshold that the impact of external electromagnetic waves must not exceed. This threshold is obtained from the tolerance threshold in terms of exposure to the electromagnetic waves (ToleranceThreshold) and the impact of the local equipment (InternalImpact).

ExternalImpact Threshold $=$ ToleranceThreshold - InternalImpact

InternalImpact $=\sum$ Iij 
where Iij is the impact of the device $j$ in the local service/application i execution

- PersonLocation: Approximate location of the sensitive person.

- Duration: Estimation of the minimum time during which the person will not change location.

These parameters were defined in such a way in order to protect the privacy of the sensitive person. Instead of communicating the exact position of the latter, the «PersonLocation» parameter will give an approximate location but sufficient to enable a reliable estimation of the impact of the external electromagnetic waves (generated by external equipment) on the sensitive person. For the same reasons, we chose to not disclose the tolerance threshold of the sensitive person. Since this parameter provides essential information for selecting the appropriate action to perform by external actors (in particular ISP), we defined a new parameter « ExternalImpactThreshold» which allows these actors to act adequately without compromising the privacy of the sensitive person.

\subsubsection{OPERATION}

When the EWER service is activated, the impact of electromagnetic waves on the sensitive person is computed at the initiation of each new service, but also periodically (period P). If the computation result indicates an exceeding of the tolerance threshold of the sensitive person, then an action can be performed. The impact computation, as well as the action selection, depends on whether the user's network is connected or not to an external network. Indeed, if the user's network has no connection to the external network (ISP), then the impact computation concerns the local electromagnetic waves and the action can only be local. Thus, the central node of the user's network does everything locally and does not need a signalling protocol. However, if the user's network is connected to the outside, the signalling protocol will be used to (i) calculate the global impact of the local and external electromagnetic waves on the sensitive person and (ii) ensure «minimal » exposure in order to protect sensitive users. Note that when launching a new service/application involving the external network, signalling will allow negotiating both QoS and security with the actors involved in this service in order to reserve the necessary resources and configure the required security mechanisms [15].

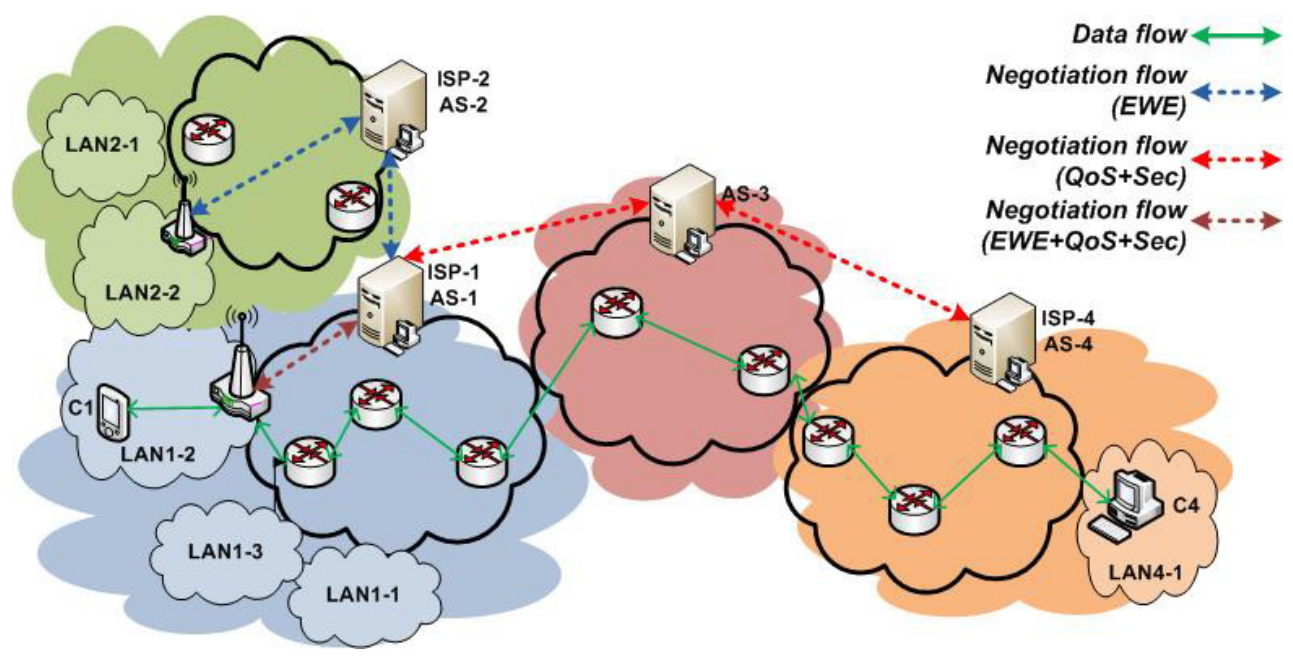

Figure 2. SLNP Signalling extended to «EWE » 
Figure 2 illustrates the new operation of the negotiation process which will be guaranteed by SLNP. Indeed, when the user's network is connected to the outside, a signalling process will be initiated at the launching of each new service / application and also periodically. When initiating a new service whose offer involves the ISP, the signalling will cover QoS and security in addition to the EWE of the sensitive person. In the other cases, the signalling will only relate to the exposure index to the electromagnetic waves, and the SLS, included in the signalling messages (Negotiate, Response, etc.), contains only the «EWE» component. When signalling includes QoS and Security, the ISP will transmit the Negotiate request (only «QoS » and «Security» components) received from its client to the other AS which are involved in the data transport. This signalling allows the different actors to agree on QoS and security levels [15]. As for the signalling related to electromagnetic radiation exposure («EWE» component), it will not necessarily imply the same network actors (AS). Therefore, the ISP will contact the relevant actors by sending them the Negotiate request (only the «EWE » component).

Thus, we note that the user's request is split in two parts at the ISP central node: "QoS + Security » on one side and «EWE » on the other side. This is due to the fact that the data path does not necessarily imply the network elements impacting the exposure index of the user (e.g. neighbouring external networks). In the same way, the two answers will be grouped together at the ISP central node. Another solution would be to distinguish two different and independent signalling processes, but we choose to group them (on the «User - ISP » segment) in order to enable the ISP to make the right decision and to avoid negative consequences regarding a criterion while satisfying another criterion. For example, changing the transmission parameters of a relay antenna connecting a user could have a negative impact on the QoS of that client's communications.

When signalling includes EWE, the first SLNP exchange (Negotiate - Response) will allow the central node of the user's network (ISP's Client) to compute the global impact of the electromagnetic waves on the sensitive person (Local equipment's impact + External equipment's impact). Then, other exchanges can take place to allow the client to ask his ISP to minimise the impact of external equipment on the sensitive person. Thus, the ISP will study the client's request (Negotiate) before replying (Response). If an ISP's action (e.g. changing the transmission parameters of a RRH) will significantly reduce the exposure level of the sensitive person, then the ISP can transmit a positive response, concerning the SLS's «EWE » component, to its client. Otherwise, the client's request will be transmitted to the other external network's impacting the sensitive user (e.g. neighbouring or remote external network's). If these external networks are also clients of the same ISP, then the request is transmitted directly.

Otherwise (WLANs impacting the sensitive user are clients of other ISPs), the ISP of the sensitive user have to go through the ISPs of the other external networks (co-operation agreements can be established between ISPs to provide good service levels for theirs clients). If several external networks are causing adverse effects on the sensitive user, then the ISP will transmit a first request to one of these networks. If this external network can not do anything or if its action does not allow the exposure index to fall below the tolerance threshold, then he can contact a second external network and so on. Note that the ISP can transmit requests starting with the external network or the ISP having the biggest impact on its client (sensitive user). This is possible beacause the information about the impact of the different networks (ISP and neighbouring LANs) is available following the first SLNP exchange. 


\section{EWER SERVICE}

In this section, we present the main features of the EWER service, which allows reducing the exposure index to electromagnetic waves related to mobile and wireless communications.

The service aiming at reducing the adverse impact of electromagnetic waves on users is intended to protect sensitive persons from the harmful impact of some electromagnetic waves. Thus, we define four main functions:

\subsection{INFORMATION}

Inform the user of the risks and propose a questionnaire to evaluate precisely his sensitivity level and create the user profile (first use). This sensitivity level, which allows computing the tolerance threshold, can be adjusted by adding objective data resulting from the measurements that will be made to provide this service appropriately. The tolerance threshold can also be adjusted following the person's request. Indeed, as soon as the sensitive person feels bad, he / she can report that via the EWER service's interface. This will result in a new computation of the tolerance threshold. Otherwise, if the sensitive person wants the precautionary principle to be applied, then the tolerance threshold takes a minimum value (Figure 3 ).

\subsection{AlerT}

The «Alert » function is activated when the whole network cannot act (or cannot act anymore) to reduce the exposure index or if its action is insufficient. In such cases, the sensitive person must act by: (i) moving to another location where its exposure level will be lower, (ii) stopping some applications, or (iii) turning off some equipment. Following an alert, the EWER service will automatically increase the exposure index computation frequency. This enables accelerating the network action cadence (reduction of the period P) in order to reduce the exposure level as quickly as possible. Moreover, after an alert, the EWER service must inform the sensitive person as soon as its exposure level returns below his tolerance threshold.

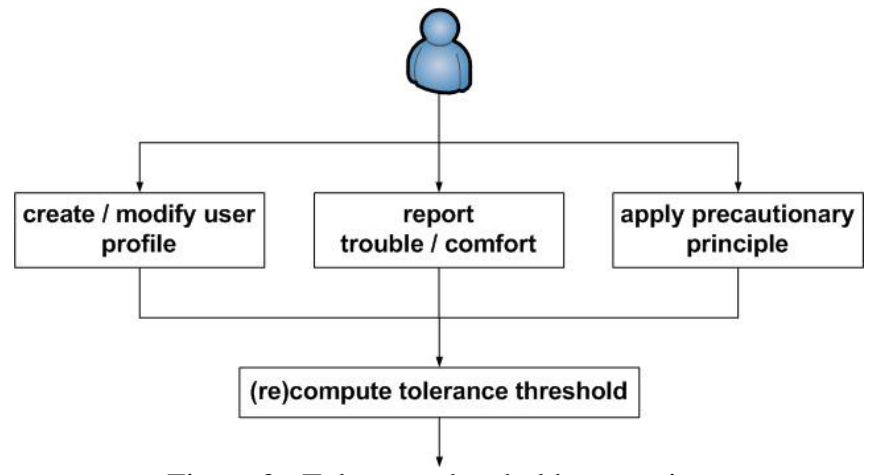

Figure 3. Tolerance threshold computing

\subsection{EXPOSURE INDEX COMPUTATION}

When the exposure index of a sensitive person can not be obtained by a measuring device that communicates with the local component of the EWER service, the EWER service must be able to compute / estimate this index. To do this, while respecting the user's privacy, we have to distribute the computation between the two main service's components: the local component (EWEM-LM: located at central device of the user's network) and the external component (EWEM-EM: located at the ISP's equipment). We note that if the user's network is not connected 
to the outside, then the exposure index computation will be performed locally. Thus, the impact of the electromagnetic waves generated by local equipment is computed using the EWEM-LM component. While, the external component EWEM-EM will be responsible for the computation of the impact of the electromagnetic waves generated by external equipment. Then, the signalling protocol will allow the ISP (EWEM-EM) to communicate the external impact to the user's network responsible node (EWEM-LM). The computation of these two impacts will be based on the available information about the location of the sensitive person as well as its radio environment. This information includes, for each radio communication source (local or external), frequency, modulation, coding, transmit power and distance to the sensitive person.

\subsection{ACTION}

This function allows the EWER service to perform actions in order to reduce the exposure index of a sensitive person. This can be done before or after exceeding the tolerance threshold. When launching a new application / service that could generate radio communications in the sensitive person's location, the EWER service will compute / estimate the person's new exposure index and act if this last could exceed the tolerance threshold. Moreover, the EWER service will measure / compute regularly (period P) the user's exposure level in order to act when this rate actually exceeds the tolerance threshold. Depending on the network environment of the sensible user, the network's action (user's network / external network) can be «impossible» or «possible». The action is called «impossible» when no action can be performed by the network to reduce the exposure index. In this case, the action plan consists in alerting the user about the tolerance threshold exceeding and transmitting a map of the surrounding electromagnetic waves and some recommendations in order to minimise his exposure level by himself. For example, the sensitive person should move to another location. The action is called "possible », if the network can actually act in order to reduce the exposure index of the sensitive person.

When the network can act, there are two types of actions:

- Local: when the electromagnetic waves impacting the user are generated by local equipment. For example, when the nodes of a local WBSN communicate information about the health status of the person;

- External: when the sources of the electromagnetic waves impacting the user are ISP's equipment or other networks (e.g. neighbouring external networks). For example, one ISP's RRH may have an impact on the sensitive person when he or she is within its coverage area. 


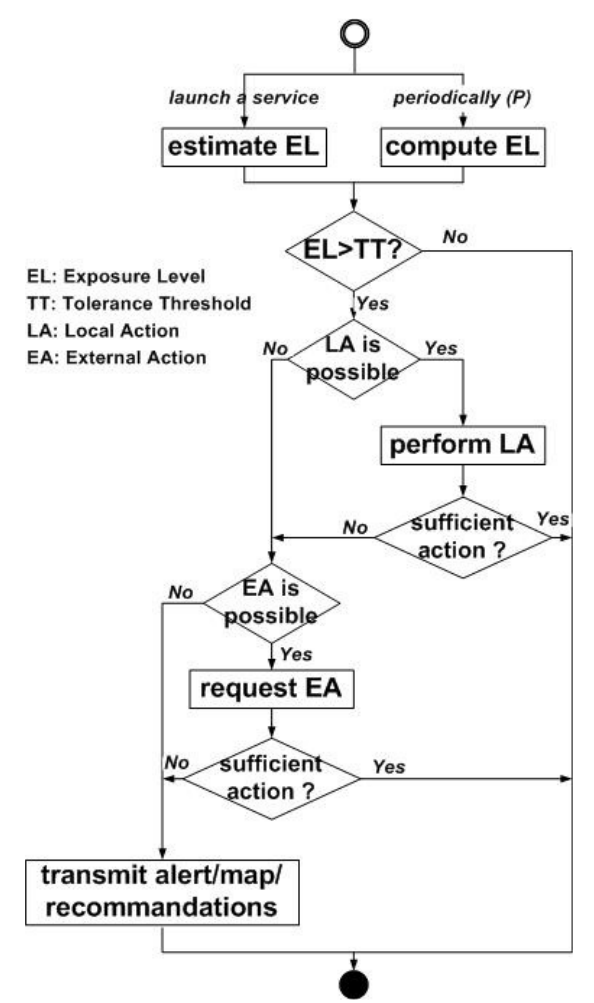

Figure 4. EWEM-LM processing

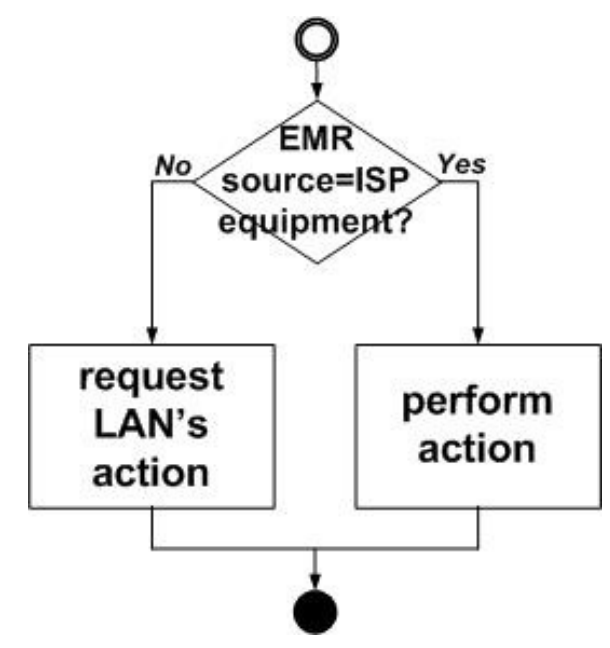

Figure 5. EWEM-EM processing

In addition, we have identified several types of actions that can be performed to reduce an individual's exposure level. These actions can be performed by the user's network or the external network:

- Modification of transmission's parameters such as frequency and power (e.g. decreasing the transmit power is an action that can be achieved rapidly) or orientation of directional antennas. This can be facilitated by the use of software radio or adaptive/smart antenna technique [8].

- Modification of the paths taken by data to avoid the area where the sensitive user is located. This can be achieved by using location-based routing algorithms.

- Reduction of the communications frequency (i.e. the number of exchanged messages), whenever possible, which can go as far as stopping communications. This can be performed by implementing mechanisms like the reasoning that we proposed to optimise the lifetime of some WSNs [17].

- Change of equipment involved in services provision. For example, in the case of a caching service for broadcasting multimedia content, we could have the choice between several devices/servers. Thus, the idea is to select the device/server that minimises the exposure of sensitive users to electromagnetic waves.

Minimising the adverse effects of electromagnetic waves, while maintaining a good quality of service level, remains a challenge. This seems to be impossible by limiting the network action to one strategy. For example, the only reduction of an antenna transmit power could create many white areas (hence the utility of SLNP). The only multiplication of relay antennas would generate an invasion by antennas and would be very expensive. The ideal would be to combine the actions 
and choose the best ones according to the situation. In addition to the actions cited above, there are other actions that may contribute to the exposure index decreasing such as Uplink and Downlink decoupling for better distribution of users within the cellular network [18] or the pooling of sites to reduce their number. Some techniques require human intervention: for example, reducing the distance between the antenna and habitations (minimum 300 to $500 \mathrm{~m}$ ), the exposure level being particularly high near the antenna. The elevation of the antenna or the reorientation of its axis will also help to reduce the exposure level of the inhabitants who were hitherto located in its main beam. Moreover, as the coverage becomes better following the elevation, it will also be possible to lower reduce the transmit power.

\section{USE-CASES}

In this section, we present two different scenarios to illustrate the utility of the service we propose (EWER). These examples will also give a concrete idea about the functioning of the service: execution, computations, signalling, etc.

\subsection{Person On The Move And Equipped With A WBSN (Environment With Significant Changes)}

In this first example, we consider a person who is telemonitored by a set of medical sensors. This person is also sensitive to the effect of electromagnetic waves. Thus, he/she is equipped with a WBSN and a smartphone acting as a gateway between the WBSN and the external network. Remember that this person could be equipped with a connected device to continuously measure its exposure level and communicate it to the smartphone. In this case, the EWER service will basically rely on these measures. However, if this type of device is not available, it is the case in this example, the service will be responsible for computing the exposure index of the sensitive person.

In this scenario, the considered person leaves a point $\mathrm{A}$ (initial position) and moves to a point $\mathrm{E}$ (final position). During its movement, it follows the path shown in figure 6 which comprises three intermediate interesting areas $(\mathrm{B}, \mathrm{C}$ and $\mathrm{D})$.

Initially, the person is at point A. The health telemonitoring service is already started. Thus, a first negotiation with SLNP has been already performed. This negotiation covered only the QoS and the security of communications with the external network (ISP). Suppose that at this moment, and before moving towards point E, the user activates the EWER service on his/her smartphone. This triggers the computation of the overall exposure level which is below the patient's tolerance threshold. As explained earlier, this computation is performed by the smartphone (exposure level to local waves computed by EWEM-LM) and the ISP (exposure rate to external waves computed by EWEM-EM). Then, using SLNP, the external network communicates the exposure level to the external waves to the smartphone. Thus, this last can obtain the overall exposure index (inferior to the patient's tolerance level). It should be noted that if this index were higher than the tolerance threshold, action would have to be taken immediately (local action and/or ISP action). We note also that if the minimum impact of the user network equipment (WBSN + smartphone) exceeds the tolerance threshold, then the action of the EWER service will in no way reach a total exposure index under the tolerance threshold. In this case, alternatives such as replacing or removing some equipment should be considered.

Then, while moving, the user passes next to « Building I » where there is a high concentration of wireless devices and antennas. This impacts the person's exposure index, which slightly exceeds his/her tolerance threshold. Indeed, given the position of these devices in the building (opposite side of the street), the impact on the person passing on the street is not very important. Following 
tolerance threshold exceed, a local action is triggered by the EWER service. This makes it possible to lower the exposure index which falls below the tolerance threshold. The action performed here consists in lowering the transmit power of some WBSN's sensors equipping the sensitive person while maintaining normal functioning of the health telemonitoring service.

When moving, the sensitive person enters the zone $\mathrm{C}$ (position $\mathrm{C} 0$ ). This time, the impact of the wireless devices and antennas of «Building II » is very important, as these, in addition to be concentrated on the street side, are much more numerous. Thus, the total exposure index (always computed following the first SLNP exchange) exceeds the tolerance threshold again. Consequently, the local component of the EWER service running on the smartphone (EWEMLM) studies the possibility of a local action. Unfortunately, no local action would allow the exposure index to fall below the tolerance threshold without adversely affecting the functioning of the health telemonitoring service. Thus, a second SLNP exchange allows the smartphone to ask the ISP if it can act on the sources of external electromagnetic waves. The ISP cannot act on any of its own equipment impacting the sensitive person. He will therefore use SLNP in order to ask the external network located at «Building II » if it can act to reduce its impact on the sensitive person passing near « Building II ». A negative response is received by the ISP, which transfers it back to the smartphone. Thus, the latter warns the sensitive person (position $\mathrm{C} 1$ ) and proposes to cross the street and go to the other side, where his/her exposure index will be lower. On the other side of the street (position C2), the patient's exposure index decreases, but unfortunately remains slightly above its tolerance threshold. The EWER service performs more frequent computations until the exposure index falls below the tolerance threshold (position $\mathrm{C} 3$ ).

Continuing on his/her way, the user crosses the field of a relay antenna located near the borrowed street. Thus, the EWER service, which runs periodically, will again detect a tolerance threshold exceed (following a SLNP exchange). Since no local action would allow this index to fall below the tolerance threshold, another SLNP exchange will take place. The SLNP request, sent by the smartphone to its ISP, will engender an action from the ISP which can reduce its transmission power during the passage time of the sensitive person. Since this action is sufficient to respect the tolerance threshold of the sensitive person, there is no need to increase the exposure index computation frequency. Note that the computation frequency depends on the movement speed as well as the configuration of the area crossed by the sensitive person. For example, computations should be more frequent in the city than in the countryside. Efficient adjusting of this frequency will allow reacting quickly to an exposure index higher than the tolerance threshold while optimising the consumed resources (CPU and bandwidth) related to the execution of the EWER service.

The current functioning of the EWER service enables reacting as quickly as possible to an exceeding of the exposure tolerance threshold of a sensitive person. Through this example, we note that the reaction of the network is not always immediate (distributed computation, SLNP signalling, decision making, etc.). Thus, the action could be actually performed when the sensitive person has already left the area where electromagnetic pollution is high. To avoid this, the functioning of the EWER service must take into account the mobility of the sensitive person that will be stored in his/her profile and updated locally. Thus, instead of acting quickly, the service will wait some time and recompute the new exposure index to see if the tolerance threshold exceeding is confirmed or not. This waiting time will be chosen according to the mobility degree of the sensitive person as well as his/her movement speed. In the case where the sensitive person is on the move, our service can be optimised in another way. Indeed, data on wave mapping can be provided to the different components of the EWER service in order to predict the evolution of the exposure level and thus act in the most effective way. 


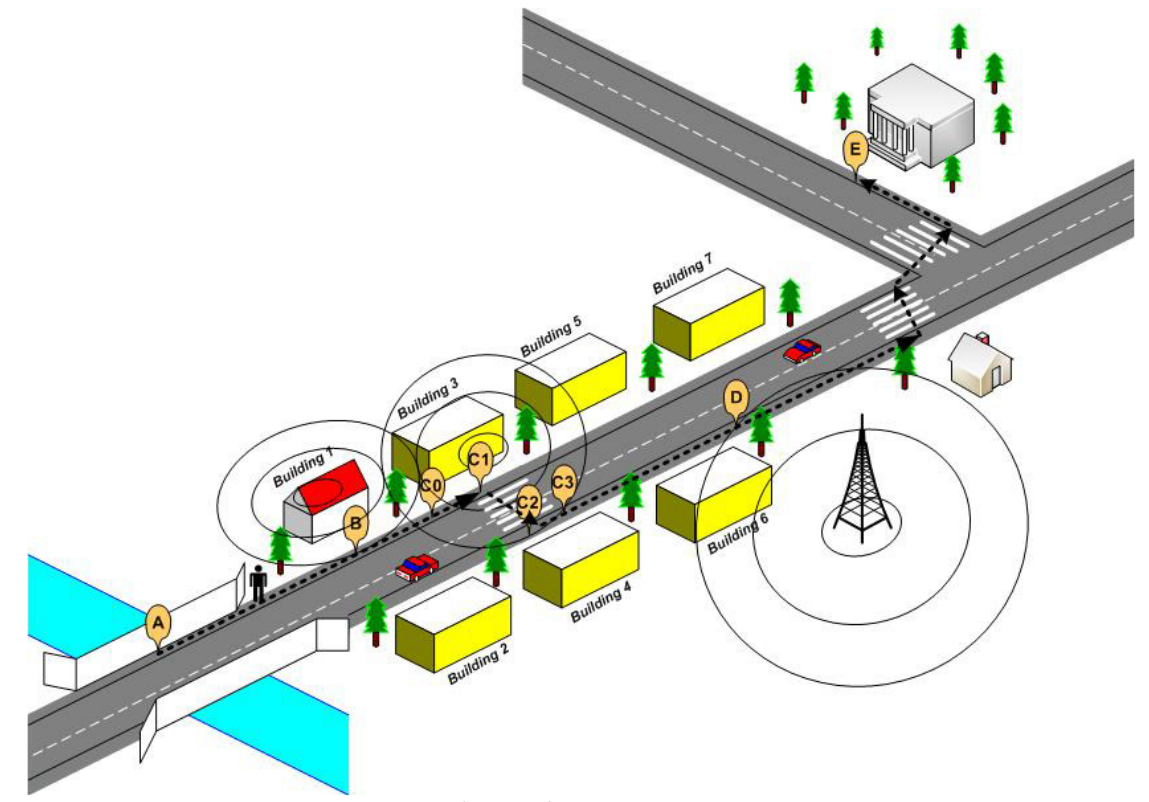

Figure 6. Use-case 1

\subsection{Person In His/Her Home (Environment With Limited Changes)}

In this example, we consider a sensitive person (tolerance threshold) wishing to apply the precautionary principle even though they have a modern habitat. This person is in his/her smart home (Home 1) providing a number of home automation services. Thus, the entourage of this person (user's network) contains: a number of wired and wireless sensors, wired and wireless terminals, some Wi-Fi antennas and a HomeBox serving as a gateway between the user's network and the external one.

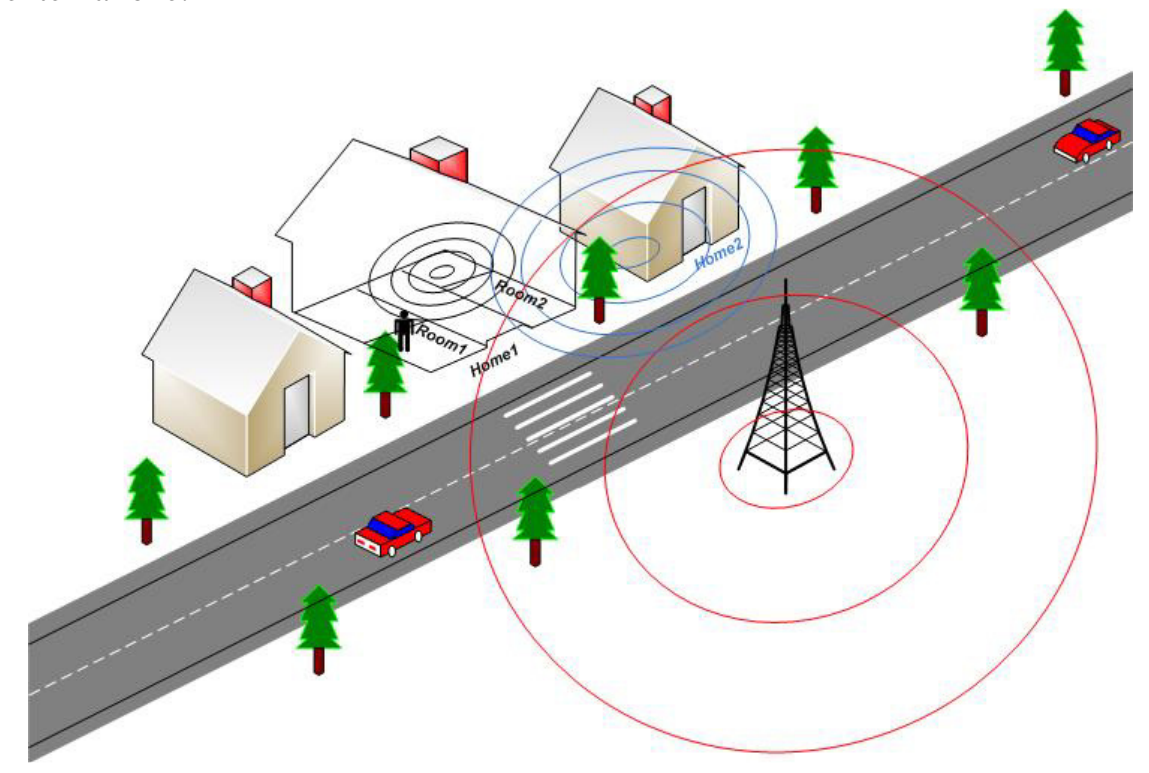

Figure 7. Use-case 2

In this scenario, the person is initially in «Room 1 » where the impact of electromagnetic waves is minimal. This is due to the important distance between that person and the vast majority of 
wireless equipment of the house. At this time, there are already some running services (home automation, multimedia, etc.) for which QoS and security have been negotiated using SLNP and configured correctly. The EWER service is also activated and the computation / estimation of the electromagnetic waves impact on the person has been performed (at the start of the services and then, with a period P) simultaneously by the HomeBox and the ISP. Indeed, as explained before, the HomeBox (EWEM-LM) has computed the impact of the local equipment (user's network) on the sensitive person. As for the ISP (EWEM-EM), it has focused on the impact of external equipment (ISPs and neighbouring LANs) on the same person. Since the total result (sum of the two impacts) is lower than the tolerance threshold reported by the person, there is no action to be taken.

Now, this person moves into the house by going into «Room 2 ». The new computation of the person's exposure index shows that, this index exceeds the tolerance threshold this time. Thus, the HomeBox starts by studying the possibility of acting locally. In order to significantly reduce the occupant exposure index, the HomeBox decides to perform two actions simultaneously: (1) lowering the transmit power towards wireless equipment in direct communication with the HomeBox and present in the person's field, (2) communicating via other wireless antennas (wired to the HomeBox) with the other wireless devices that are no longer within range following action 1. Following these two actions, the exposure index falls below the tolerance threshold.

Sometime later, this rate (computed periodically) exceeds the tolerance threshold again, despite the unchanged position of the sensitive person (still in « Room 2 »). This is due to the increase of the external impact due to the electromagnetic waves generated by the wireless communications of the neighbouring LAN's equipment (located in a neighbouring house: « Home $2 »$ ). As usual, HomeBox investigates the possibility of a local action. Unfortunately, no local action could be taken without causing major degradation of the running applications (home automation, multimedia, etc.). Thus, a second SLNP exchange (the first exchange always allows computing the total exposure index) will allow the HomeBox to ask the ISP to act on external sources in order to reduce the person's exposure index and to pass it below the tolerance threshold. The ISP begins by acting on its equipment which has an impact on the exposure index of the sensitive person. Indeed, in this example, the ISP can reduce the transmit power of one of its relay antennas which is close to the house occupied by that person (Home 1). The transmit power reduction is performed as far as possible, because it should not cause a significant degradation in the services provided to other ISP's clients. In this example, the ISP's action allows reducing slightly the exposure level which therefore remains above the tolerance threshold.

Thus, the ISP must transmit the request of the client benefiting from the EWER service to the neighbouring external network (Home 2). In this example, this external network (Home 2) is a client of the same ISP. Thus, the ISP will directly ask if it can act in order to reduce its impact on the sensitive person occupying the «Home $1 »$. This is done by transmitting a SLNP request including only the EWE component. Note that if the external network located in «Home 2 » was not also a client of the same ISP, then the latter would transmit the request (SLNP) via the ISP of the neighbouring external network. In this scenario, the LAN located in « Home 2 » cannot act. It then replies negatively to the received request. This response will be transmitted to the HomeBox of the sensitive person occupying «Home 1 ». Since no new action can be performed by the networks (user's and external) to reduce the exposure index of the sensitive person, the HomeBox must alert the latter. Thus, the sensitive person can reduce its exposure index by: (1) moving to another room where there are fewer waves, or (2) disabling some applications (or even all applications). This could be done by suspending or turning off wireless sensors, devices and antennas. On the other hand, if the external electromagnetic waves have an important and permanent impact on the sensitive person, then it must consider moving or making important adjustments such as shielding his house to transform it into a cage of Faraday (see section 2.1.2.2). 


\section{Conclusion And Perspectives}

In the context of electromagnetic waves explosion, we propose an innovative service. This service will allow sensitive persons (children, patients with medical devices, electro-hypersensitive persons, etc.) to have a minimum exposure level to electromagnetic waves or, at least, an exposure level respecting their tolerance thresholds. The proposed service can act on electromagnetic waves related to network communications (e.g. Bluetooth, Wi-Fi, WiMax, UMTS, etc.) in different ways (e.g. change of transmission parameters, change of routing paths, etc.). In this paper, we have detailed the different functions (information, alert, exposure index computation and action) of the proposed service as well as the defined mechanisms (signalling, computation and action modules, etc.).

This complete and detailed proposal has not yet been implemented. Therefore, the next step in our work is to implement the EWER service to validate its operation. Implementation of our proposal will also allow us to evaluate the performance of the proposed approach such as reaction time, success rate in various contexts, etc. In order to improve the operation of the proposed service, further improvements and extensions can be proposed. Among these improvements, we mention the consideration of the user's mobility which could be achieved by implementing an efficient prediction mechanism for example. This will contribute to the effectiveness of the actions performed by the EWER service in a non-stable radio environment. Another situation could complicate the issue dealt with in this paper. It consists in considering two or more sensitive people in the same user's network (e.g. home network). In general, scaling up should also be considered. We will also consider the integration of this service into a $5 \mathrm{G}$ architecture by placing the necessary intelligence as close as possible to enable the ISP and the access point to act as quickly as possible [19]. It will be also very interesting to enable an easy integration of this service in eHealth systems [20], [21].

\section{REFERENCES}

[1] Third Generation Partnership Project 2, Available online: http://www.3gpp2.org/

[2] European Commission, "Europe 2020". Available online: http://ec.europa.eu/

[3] H.S. Aboul Ezz, Y.A. Khadrawy, N.A. Ahmed, N.M. Radwan, M.M. El Bakry, "The effect of pulsed electromagnetic radiation from mobile phone on the levels of monoamine neurotransmitters in four different areas of rat brain”, European Review for Medical and Pharmacological Sciences, 2013

[4] K.K. Kesari, S. Kumar, J. Behari, "Effects of Radiofrequency Electromagnetic Wave Exposure from Cellular Phones on the Reproductive Pattern in Male Wistar Rats", Journal of Applied Biochemistry and Biotechnology, 2011

[5] S. MANN, "Assessing personal exposures to environmental radiofrequency electromagnetic fields" C. R. Physique, 2010

[6] Y.A. Sambo, F. Héliot, M.A. Imran, "A Survey and Tutorial of Electromagnetic Radiation and Reduction in Mobile Communication Systems”, IEEE Communication Surveys \& Tutorials, Vol. 17, $\mathrm{n}^{\circ} .2,2015$

[7] J. Palicot, C. Roland, "On the use of Cognitive Radio for Decreasing Electromagnetic Radiation", URSI 05, XXVIII General Assembly, New Delhi, India, October 23-29, 2005

[8] LEXNET project, "Low EMF Exposure Future Networks", Available online: www.lexnet.fr/

[9] P. Staebler, "Human Exposure to Electromagnetic Waves", "Collection Ondes", November 2016, iste editions

[10] EHS \& MCS Research and Treatment European Group, "How to protect yourself when you become electrohypersensitive (EHS)", Available online: http://www.ehs-mcs.org/

[11] World Health Organization, "Electromagnetic fields (EMF) - What are electromagnetic fields ? Summary of health effects" Available online:

http://www.who.int/peh-emf/about/WhatisEMF/en/index 1.html 
[12] French Republic - Légifrance, French Republic, "Law n 2015-136 of 9 February 2015 on sobriety, transparency, information and consultation on exposure to electromagnetic waves", available online: https://www.legifrance.gouv.fr/eli/loi/2015/2/9/2015-136/jo/texte

[13] Electrosmog, "EMF Measuring devices (Appareils de mesure de CEM)", Available online: www.electrosmog.info/IMG/pdf/Appareils-Mesure.pdf

[14] Electrosmog, "Mobile phones and EMF (Téléphones mobiles et champs électromagnétiques)", Available online: http://www.electrosmog.info/IMG/pdf/Telephones-Mobiles.pdf

[15] M.A. Chalouf and F. Krief, "A secured, automated, and dynamic end-to-end service level negotiation", Concurrency and Computation: Practice and Experience, Volume 25, Number 1, January 2013, pp. 180-202

[16] Mushtaq Ahmed Bhat and Vijay Kumar, «Calculation of SAR and Measurement of Temperature Change of Human Head Due To The Mobile Phone Waves At Frequencies $900 \mathrm{MHz}$ and $1800 \mathrm{MHz}$ », in Advances in Physics Theories and Applications, Vol.16, 2013, www.iiste.org

[17] M. Mbaye, M.A. Chalouf, F. Krief, M. Peres, "Real-World Deployment of a Smart and Green Wireless Sensor Network for Intrusion Detection”, International Journal of Computer Science Issues, IJCSI, Vol. 12, Issue 6, December 2015.

[18] F. Boccardi, J. Andrews, H. Elshaer, M. Dohler, S. Parkvall, P. Popovski, S. Singh, "Why to decouple the Uplink and Downlink in Cellular Networks and How to Do It", IEEE Communications Magazine 2016, Volume: 54, Issue: 3, pp. 110 - 117

[19] Thierry Coupaye, "Fog Computing et Geo-distributed Cloud", Research Blog, Orange Labs, available online: https://recherche.orange.com/fog-computing-et-cloud-geo-reparti/

[20] O. Hamdi, M. A. Chalouf, D. Ouattara, F. Krief, "eHealth: Survey on research projects, comparative study of telemonitoring architectures and main issues", Journal of Network and Computer Applications, 2014, pp. 100-112.

[21] Amna Abdullah, Asma Ismael, Aisha Rashid, Ali Abou-Elnour and Mohammed Tarique, "Real Time Wireless Health Monitoring Application Using Mobile Devices", International Journal of Computer Networks \& Communications, IJCNC, May 2015, Volume 7, Number 3.

\section{AuTHORS}

Mohamed-Aymen Chalouf received his Ph.D. from the University of Bordeaux 1. Currently, he is Asssociate Professor at the University of Rennes 1 (IUT of Lannion) and member of the IRISA (Institut de Recherche en Informatique et Systèmes Aléatoires) Laboratory, «Networks, Telecommunications and Services» department (D2). His research interests include the management of quality, security and energy in new network architectures, systems and services. His research has produced a number of publications in international journals and conferences.

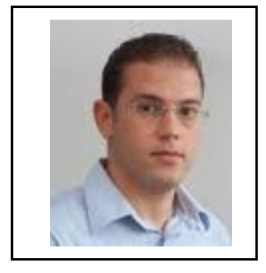

Francine KRIEF obtained the HDR degree (Habilitation à Diriger des Recherches) at University of Paris 6 on Context-aware Management, in December 2003. Currently, she is Professor at Bordeaux-INP and member of CNRS LaBRI Laboratory, UMR 5800, "Programming, Networks and Systems" team. Her main research activities concern self-management for wired and wireless networks, endto-end signaling protocols and green networking. Her work on network and service management has led to many publications in journals and at conferences.

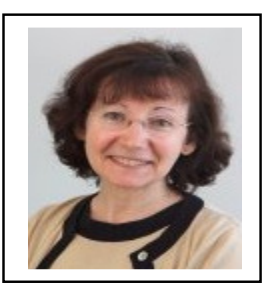

Dramane Ouattara is, currently, Assistant Lecturer in Computer Science at University of Korhogo in Côte d'Ivoire. During 2014-2016, he was a Rresearch and Teaching Assistant at Bordeaux University and Bordeaux INP in France. He received his Ph.D. in computer science from Bordeaux University in 2014. His research interests span both computer networking and network science that includes cognitive radio networks. Much of his work has been focused on improving ehealth communications by using cognitive radio systems. Currently, he is interested in the problems of electromagnetic waves and their effects on the

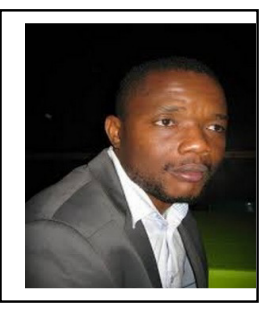
population. 